\title{
Prediction of Peptide Conformation by the Multicanonical Algorithm ${ }^{1}$
}

\author{
Ulrich H.E. Hansmann ${ }^{2}$ and Yuko Okamoto ${ }^{3}$
}

\begin{abstract}
We test the effectiveness of the multicanonical algorithm for the tertiary structure prediction of peptides and proteins. As a simple example we study Metenkephalin. The lowest-energy conformation obtained agrees with that determined by other methods such as Monte Carlo simulated annealing. But unlike to simulated annealing the relationship to the canonical ensemble remains exactly controlled. Thermodynamic quantities at various temperature can be calculated from one run.
\end{abstract}

A protein or a peptide is a molecule that consists of a chain of $N$ amino acid residues. There are 20 different amino acids known in nature. When $N$ is large one calls the molecule a protein, otherwise a peptide. The prediction of tertiary structures of proteins, which determine their biological function, from their primary sequences remains one of the long-standing unsolved problems (for recent reviews, see, for example, Refs. [1]). It is widely believed that this structure corresponds to the global minimum in the energy. So the problem amounts to finding the global minimum energy out of a huge number of local minima separated by high tunneling barriers. Within the presently available computer resources, the traditional methods such as molecular dynamics and Monte Carlo simulations at relevant temperatures tend to get trapped in local minima. One of the methods which which seem to alleviate this multiple-minima problem is simulated annealing. [2] However, a disadvantage of simulated annealing is that there is no established protocol for annealing and a certain number (which is not known a priori) of runs are necessary to evaluate the performance. Moreover, the relationship of the obtained conformations to the equilibrium canonical ensemble at a fixed temperature remains unclear.

These problems may be overcome by the multicanonical algorithm which was recently proposed by Berg et al. [3] Originally developed to overcome the supercritical slowing down of first-order phase transitions, [4] it has also been tested for systems with conflicting constraints such as spin glasses. [5, 6, 7] The latter systems suffer from a similar multiple-minima problem and it was claimed that the multicanonical algorithm outperforms simulated annealing in these cases. [6]

\footnotetext{
${ }^{1}$ To appear in the Proceedings of the Sixth Annual Workshop on Recent Developments in Computer Simulation Studies in Condensed Matter Physics, 22-26 Feb. 1993, Athens, Georgia.

${ }^{2}$ Department of Physics and Supercomputer Computations Research Institute (SCRI), The Florida State University, Tallahassee, FL 32306, USA.

${ }^{3}$ Department of Physics, Nara Women's University,Nara 630, Japan.
} 
The idea of this method is based on performing Monte Carlo simulations in a multicanonical ensemble 3, 8 instead of the usual (canonical) Gibbs-ensemble. In the canonical ensemble, configurations at an inverse temperature $\hat{\beta} \equiv 1 / R T$ are weighted with the Boltzmann factor $\mathcal{P}_{B}(E)=\exp (-\hat{\beta} E)$. The resulting probability distribution is given by

$$
P_{B}(E) \propto n(E) \mathcal{P}_{B}(E)
$$

where $n(E)$ is the spectral density. In the multicanonical ensemble, [3, 8 on the other hand, the probability distribution is defined in such a way that a configuration with any energy enters with equal probability:

$$
P_{m u}(E) \propto n(E) \mathcal{P}_{m u}(E)=\text { const. }
$$

Then it follows that the multicanonical weight factor should have the form

$$
\mathcal{P}_{m u}(E) \propto n^{-1}(E) .
$$

In order to define a explicit form of this weight factor, we introduce two parameters $\alpha(E)$ and $\beta(E)$ as follows: [3]

$$
\mathcal{P}_{m u}(E)=\exp \{-(\hat{\beta}+\beta(E)) E-\alpha(E)\} .
$$

For any fixed $\beta(E)$ and $\alpha(E)$ this leads to the canonical weight factor with the inverse temperature $\beta=\hat{\beta}+\beta(E)$, hence the name "multicanonical". For a numerical simulation one needs estimators for the multicanonical parameters $\beta(E)$ and $\alpha(E)$. The iterative procedure by which one can get such estimators is described elsewhere. [9] Once the multicanonical parameters are determined, one multicanonical run is in principle enough to calculate all thermodynamic quantities by re-weighting. 10] Since in the multicanonical ensemble all energies enter with equal probability a simulation may overcome the barriers between local minima by connecting back to the high temperature states. In this way the global minimum can be explored.

In the present work we apply the multicanonical algorithm to the problem of protein folding, the tertiary structure prediction of peptides and proteins. The purpose of this work is primarily to test the effectiveness of the algorithm. For this reason we have studied one of the simplest peptide, Met-enkephalin. The lowest-energy conformation for the potential energy function ECEPP/2 [1] is known [12] and analyses with Monte Carlo simulated annealing with ECEPP/2 also exist. 13, 14]

Met-enkephalin has the amino-acid sequence Tyr-Gly-Gly-Phe-Met. For our simulations the backbone was terminated by a neutral $\mathrm{NH}_{2}-$ group at the $\mathrm{N}$-terminus and a neutral - COOH group at the C-terminus as in the previous works of Met-enkephalin. [12] The potential energy function that we used is given by the sum of the electrostatic term $E_{e s}$, the van der Waals energy $E_{v d W}$, and hydrogen-bond term $E_{h b}$ for all pairs of atoms in the peptide together with the torsion term $E_{\text {tors }}$ for all torsion angles:

$$
\begin{aligned}
E_{t o t} & =E_{e s}+E_{v d W}+E_{h b}+E_{t o r s} \\
E_{e s} & =\sum_{(i, j)} \frac{332 q_{i} q_{j}}{\epsilon r_{i j}}
\end{aligned}
$$




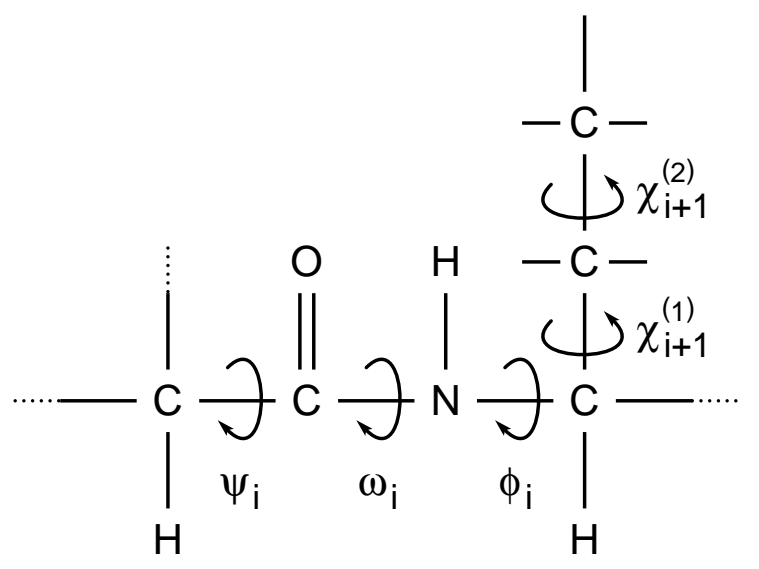

Figure 1: Definition of dihedral angles
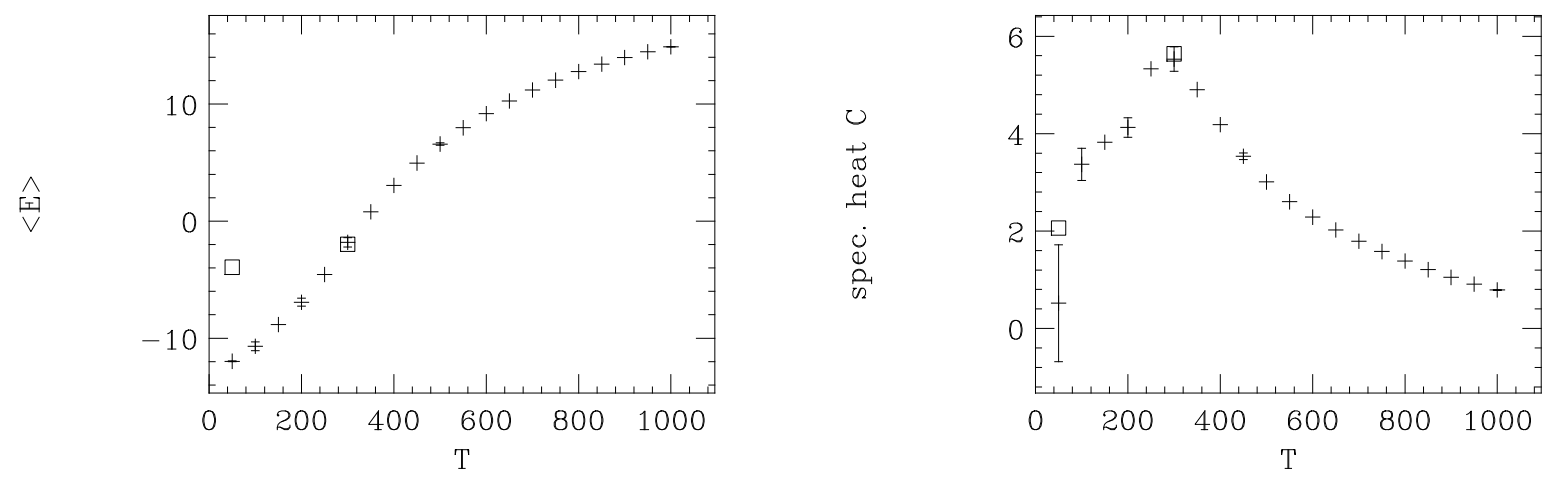

Figure 2: Average energy $\langle E>$ and specific heat $C$ of Met-enkephalin as a function of temperature evaluated by multicanonical algorithms. The results of canonical simulations at fixed temperatures $(50 \mathrm{~K}$ and $300 \mathrm{~K})$ are also plotted $(\square)$.

$$
\begin{aligned}
E_{v d W} & =\sum_{(i, j)}\left(\frac{A_{i j}}{r_{i j}^{12}}-\frac{B_{i j}}{r_{i j}^{6}}\right), \\
E_{h b} & =\sum_{(i, j)}\left(\frac{C_{i j}}{r_{i j}^{12}}-\frac{D_{i j}}{r_{i j}^{10}}\right), \\
E_{\text {tors }} & =\sum_{l} U_{l}\left(1 \pm \cos \left(n_{l} \alpha_{l}\right)\right) .
\end{aligned}
$$

$r_{i j}$ is the distance between the atoms $i$ and $j$, and $\alpha_{l}$ is the torsion angle for the chemical bond $l$. For a definition of these angles which represent the true degrees of freedom see Fig. [1. The parameters $\left(q_{i}, A_{i j}, B_{i j}, C_{i j}, D_{i j}, U_{l}\right.$ and $\left.n_{l}\right)$ for the energy function were adopted from ECEPP $/ 2$, [11]. The effect of surrounding atoms of water is neglected and the dielectric constant $c$ is set equal to 2. The computer code KONF90, [15 was modified to accommodate the multicanonical method. The peptide-bond dihedral angles $\omega$ were fixed at the value $180^{\circ}$ for simplicity, which leaves 19 angles $\phi_{i}, \Psi_{i}$ and $\chi_{i}$ as independent variables.

In Fig. 2a we show the average energy, obtained by our method in a run with $10^{5}$ 
Table 1: Table I. Energy and dihedral angles of the lowest-energy conformations of Metenkephalin obtained by multicanonical runs. Conformation A is the lowest-energy conformation obtained by Monte Carlo simulated annealing (taken from Ref. 13).

\begin{tabular}{|c|r|r|r|r|r|r|r|}
\hline Conformation & \multicolumn{1}{|c|}{$\mathrm{A}$} & \multicolumn{1}{c|}{1} & \multicolumn{1}{c|}{2} & \multicolumn{1}{c|}{3} & \multicolumn{1}{c|}{4} & \multicolumn{1}{c|}{5} & 6 \\
\hline $\mathrm{E}$ [ kcal/mol ] & -11.9 & -11.9 & -12.0 & -12.0 & -12.1 & -12.0 & -11.9 \\
\hline$\phi_{1}$ & 98 & 90 & 91 & 90 & 97 & 96 & 98 \\
$\psi_{1}$ & 154 & 153 & 152 & 154 & 151 & 153 & 156 \\
$\phi_{2}$ & -161 & -160 & -157 & -161 & -158 & -161 & -163 \\
$\psi_{2}$ & 69 & 72 & 64 & 71 & 71 & 68 & 65 \\
$\phi_{3}$ & 65 & 64 & 66 & 63 & 64 & 64 & 66 \\
$\psi_{3}$ & -93 & -95 & -92 & -95 & -94 & -89 & -92 \\
$\phi_{4}$ & -85 & -82 & -80 & -77 & -83 & -85 & -80 \\
$\psi_{4}$ & -27 & -26 & -29 & -32 & -30 & -31 & -29 \\
$\phi_{5}$ & -83 & -81 & -82 & -78 & -80 & -82 & -86 \\
$\psi_{5}$ & 142 & 142 & 138 & 137 & 145 & 151 & 147 \\
$\chi_{1}^{1}$ & -179 & 179 & -177 & 179 & 179 & -178 & -176 \\
$\chi_{1}^{2}$ & -112 & -110 & -117 & -109 & -111 & -115 & -114 \\
$\chi_{1}^{3}$ & 149 & 144 & 146 & 143 & 149 & 145 & 142 \\
$\chi_{4}^{1}$ & 180 & -176 & 178 & 177 & 180 & -178 & 180 \\
$\chi_{4}^{2}$ & 73 & 79 & 81 & 86 & 79 & 78 & 78 \\
$\chi_{5}^{1}$ & -65 & -64 & -67 & -67 & -66 & -67 & -66 \\
$\chi_{5}^{2}$ & 180 & -179 & 180 & 180 & -176 & 180 & 176 \\
$\chi_{5}^{3}$ & 179 & 178 & 179 & -179 & -179 & -178 & -178 \\
$\chi_{5}^{4}$ & -55 & -66 & -59 & -62 & -61 & -60 & -57 \\
\hline
\end{tabular}

sweeps, as a function of temperature. The value $\approx-12 \mathrm{kcal} / \mathrm{mol}$ at $T=50 \mathrm{~K}$ is very close to the global-minimum energy obtained by other methods. 112, 13, 14] In Fig. 2b we likewise present the "specific heat" (per residue), which is defined by

$$
C=\beta^{2} \frac{<E^{2}>-<E>^{2}}{5} .
$$

It has a peak around $T=300 \mathrm{~K}$, which indicates that this temperature is important for peptide folding.

During the production run the system reached the global-energy minimum region six times. The lowest-energy conformation within each visit is listed in Table I together with the global-minimum energy conformation (Conformation A in Table I) obtained by simulated annealing. 113] Conformations 1-6 are the results at Monte Carlo steps 20128, 39521, 44462, 65412, 89413, and 95143. Hence, the system reached the lowest-energy region in every 5000 to 20000 Monte Carlo steps. The energies are almost all equal, and the lowest-energy value in the present work $(-12.1 \mathrm{kcal} / \mathrm{mol})$ is slightly less than the previous result $(-11.9 \mathrm{kcal} / \mathrm{mol})$ by simulated annealing. 13. Most of the dihedral angles of the six conformations also agree with the corresponding ones of Conformation A within $\approx 5^{\circ}$.

We have applied the recently developed multicanonical algorithm to the problem of predicting the peptide conformation. This method avoids getting trapped in a local min- 
imum of energy function by connecting back to high temperature states and enhances in this way the probability to find the global minimum. We have demonstrated the effectiveness of the algorithm by reproducing the lowest-energy conformation of Met-enkephalin. Furthermore, the multicanonical algorithm can yield various thermodynamic quantities as a function of temperature from only one production run.

\section{Acknowledgements:}

Our simulations were performed on the SCRI cluster of fast RISC workstations. This work is supported, in part, by the Department of Energy, contract DE-AC03-76SF00515, DEFG05-87ER40319, DE-FC05-85ER250000 and by the Deutsche Forschungsgemeinschaft under contract H180411-1. Y.O likes to thank SCRI for the kind hospitality extended to him during a visit.

\section{References}

[1] M. Levitt, Curr. Opin. Struct. Biol., 1, 224 (1991).

[2] S. Kirkpatrick, C.D. Gelatt, Jr., and M.P. Vecchi, Science, 220, 671 (1983).

[3] B.A. Berg and T. Neuhaus, Phys. Lett., B267, 249 (1991).

[4] B.A. Berg and T. Neuhaus, Phys. Rev. Lett., 68, 9 (1992); B. Berg, U. Hansmann and T. Neuhaus, Phys. Rev. B 47 (1993) 497; B. Berg, U. Hansmann and T. Neuhaus, Z. Phys. B 90 (1993) 229; W. Janke, B. Berg and M. Katoot, Nucl. Phys., B382 649 (1992);

[5] B. Berg and T. Celik, Phys. Rev. Lett., 692292 (1992).

[6] B. Berg and T. Celik, Int. J. Mod. Phys. C 3 (1992) 1251.

[7] B. Berg, T. Celik and U. Hansmann, to appear in Europhysics Letters.

[8] G.M. Torrie and J.P. Valleu, J. Comp. Phys., 23, 187 (1977).

[9] U. Hansmann and Y. Okamoto, FSU-SCRI-93-12, submitted to J. Comp. Chem.

[10] A.M. Ferrenberg and R.H. Swendsen, Phys. Rev. Lett., 61, 2635 (1988); 63 , 1658(E) (1989), and references given in the erratum.

[11] F.A. Momany, R.F. McGuire, A.W. Burgess, and H.A. Scheraga, J. Phys. Chem., 79, 2361 (1975); G. Némethy, M.S. Pottle, and H.A. Scheraga, J. Phys. Chem., 87, 1883 (1983); M.J. Sipple, G. Némethy, and H.A. Scheraga, J. Phys. Chem., 88, 6231 (1984).

[12] Z. Li and H.A. Scheraga, Proc. Natl. Aca. Sci., U.S.A., 84, 6611 (1987); A. Nayeem, J. Vila, and H.A. Scheraga, J. Comp. Chem., 12, 594 (1991).

[13] Y. Okamoto, T. Kikuchi, and H. Kawai, Chem. Lett., 1992, 1275.

[14] B. von Freyberg and W. Braun, J. Comp. Chem., 12, 1065 (1991).

[15] H. Kawai, Y. Okamoto, M. Fukugita, T. Nakazawa, and T. Kikuchi, Chem. Lett., 1991, 213; Y. Okamoto, M. Fukugita, T. Nakazawa, and H. Kawai, Protein Eng., 4, 639 (1991). 